\title{
TIEMPO, TRABAJO Y VIDA COTIDIANA EN EL CONURBANO BONAERENSE, ARGENTINA
}

\section{TIME, WORK AND DAILY LIFE IN THE BUENOS AIRES, ARGENTINA}

\author{
María Claudia Cabrera* \\ Malena Victoria Hopp**
}

\begin{abstract}
RESUMEN
El objetivo de este artículo es explorar cómo se (re)produce la desigualdad a través de la organización del tiempo, según las distintas formas de inserción laboral de las personas. Con ese objetivo, se realiza un estudio cuantitativo con base en una encuesta realizada en el año 2009, a trabajadores mayores de 18 años, residentes en Partidos del Gran Buenos Aires (Argentina). Se parte de la hipótesis según la cual, las diversas formas de distribución y organización del tiempo pueden considerarse como expresiones de condiciones de desigualdad.
\end{abstract}

PALABRAS CLAVE: ARGENTINA * TIEMPO * TRABAJO * DESIGUALDAD

\section{ABSTRACT}

The objective of this article is to explore how the inequality is produced through the organization of the time, according to the different forms of labor insertion. The hypothesis shown here is that the diverse forms of distribution and organization of time can be considered as expressions of inequality conditions. The study was quantitative, on the basis of a survey implemented in 2009 to workers majors of 18 years, residents in Parties of Buenos Aires (Argentina).

KEYWORDS: ARGENTINA * TIME * WORK * INEQUALITY

Grupo de Estudios sobre Política Social y Condiciones de Trabajo, Instituto Gino Germani, Universidad de Buenos Aires, Argentina (UBA). mccabrera@gmail.com
** Grupo de Estudios sobre Política Social y Condiciones de Trabajo, Instituto Gino Germani, Universidad de Buenos Aires, Argentina (UBA). malenahopp@yahoo.com.ar 


\section{INTRODUCCIÓN}

Este artículo presenta los primeros resultados de una investigación más amplia que se propone analizar la relación entre la forma en que se erigen $y$ diluyen las fronteras que demarcan el tiempo de trabajo y el tiempo de la vida, y su correspondencia con la (re)producción de desigualdades. Se parte de la hipótesis, según la cual las diversas formas de distribución y organización del tiempo de trabajo y del tiempo "propio", pueden considerarse como uno de los modos en que se expresan condiciones de desigualdad. Tomando en consideración esa hipótesis, interesa conocer los modos en que las cuestiones vinculadas a la vida social, familiar $y$ doméstica se "filtran" en el tiempo formalmente dedicado al trabajo.

En cuanto a la metodología utilizada, el artículo recurre a los resultados de una encuesta realizada entre los meses de octubre y noviembre de 2009 , cuya unidad de observación fueron trabajadores mayores de 18 años, residentes en Partidos del Gran Buenos Aires ${ }^{1}$.

La exposición de los resultados se organiza del siguiente modo: en primer lugar, se desarrollan los fundamentos de la hipótesis, luego se describe el enfoque teórico-metodológico utilizado y los criterios de construcción del "índice de labilidad" de las fronteras entre el tiempo de la vida y el tiempo del trabajo, que permitió indagar acerca de los modos en que las cuestiones vinculadas a la esfera social, familiar y doméstica se "filtran" en el tiempo formalmente dedicado al trabajo; además el "índice de participación doméstica", a través del cual se puede conocer las diversas posiciones que ocupan los sujetos en el hogar y el tiempo que le dedican a las tareas reproductivas, es decir sus condiciones de participación doméstica. Posteriormente, se describe los modos en que se establece la relación entre tiempo de vida $y$ de trabajo, las tensiones $y$ conflictos que allí aparecen, vinculándolas con el tipo de actividad, las condiciones físicas y organizativas

1 El Instituto Nacional de Estadísticas y Censos (INDEC) incluye en esta denominación a los 24 partidos de la provincia de Buenos Aires que conforman el Gran Buenos Aires, sin la Ciudad de Buenos Aires. en las que se realiza el trabajo y las condiciones de participación doméstica de los sujetos. Por último, se compara las posibilidades de organización del tiempo de distintos grupos sociales, a fin de analizar las formas de desigualdad que la relación con el tiempo plantea.

2. TIEMPO Y DESIGUALDAD: LA CONSTRUCCIÓN SOCIAL DE LAS FRONTERAS ENTRE MUNDO DEL TRABAJO Y VIDA COTIDIANA

La importancia del tiempo en la construcción de la vida social ha sido señalada tempranamente por las ciencias sociales. Cabe recordar que para Durkheim, las categorías espacio-temporales son construcciones colectivas de central importancia que expresan una determinada concepción común acerca de los ritmos de la vida social.

Lo propio del desarrollo de las sociedades capitalistas, ha sido la progresiva pero firme construcción de dos esferas diferenciadas que comprendían tareas específicas — productivas $y$ reproductivas $-y$ delineaban dos temporalidades: el tiempo destinado al trabajo y el tiempo propio. De este modo, la regularización del trabajo asalariado cambió la relación con el tiempo, definiendo un espacio exclusivamente destinado al trabajo (Thompson, 1989). A diferencia del desarrollo del capitalismo en los países centrales, en América Latina históricamente han coexistido formas de producción modernas, en las que dicha separación se delimitó más marcadamente, con el desarrollo de economías informales y/o de subsistencia en las que los tiempos $y$ espacios de la vida familiar $y$ de la producción se encontraban superpuestos.

Ahora bien, si lo propio del capitalismo del siglo Xx fue la distinción entre ambas esferas -incluso en nuestra región, aunque con las particularidades mencionadas-, las profundas transformaciones socio-laborales y productivas de las últimas décadas, supusieron una reconfiguración de sus límites (Castel, 2004; Boltanski y Chiapello, 2002; Sennet, 2000). Esta transformación comprende diversos aspectos: nuevas condiciones de producción en el marco de la globalización, el uso de nuevas tecnologías de comunicación, las reglamentaciones laborales 
flexibles y la instrumentación de formas de gestión del trabajo basadas en la extrema implicación personal (Luci, 2009). Transformaciones de distintos órdenes redefinen así, no solamente los espacios dedicados a las actividades laborales, sino la construcción social del tiempo que las separa de aquellas consideradas como "privadas" y que hacen referencia al goce de un "mundo de la vida" por fuera del trabajo (Grassi y Danani, 2009).

Sin embargo, estas transformaciones en la organización del trabajo, que sin duda posibilitan nuevas relaciones entre tiempo de trabajo $y$ de vida, no se han producido del mismo modo y con la misma intensidad en todos los países o regiones y como se verá más adelante, tampoco abarcan la totalidad de formas laborales que integran el mundo del trabajo. Al respecto, se considera que la labilidad o rigidez de las fronteras entre trabajo $y$ vida pone de manifiesto las distintas posibilidades que tienen los sujetos de organizar y/o disponer de su tiempo, en función de sus obligaciones laborales y necesidades de la vida doméstica.

\subsection{TIEMPO Y DESIGUALDAD}

El problema de la desigualdad ha sido abordado desde distintas perspectivas. Algunas conceptualizaciones se centraron en dar cuenta de la desigualdad existente entre personas y grupos sociales, según su situación frente a la posesión de los medios de producción y la capacidad de control del proceso productivo. Las mediciones estadísticas que derivan de esta mirada centrada en el proceso de producción económica y la división del trabajo, se enfocaron en el análisis de las categorías ocupacionales $y$ del nivel de ingresos con ellas vinculadas ${ }^{2}$. Esta perspectiva permite dar cuenta de las desigualdades entre distintas categorías sociales, que siguiendo a Fitoussi y Rosanvallon (2003), se pueden definir como desigualdades estructurales o "tradicionales".

Otras perspectivas incorporan nuevas dimensiones de análisis al problema de la

$2 \quad$ Desde esta perspectiva, el indicador de desigualdad dominante es el coeficiente de Gini, el cual mide la desigualdad en relación al nivel de ingresos. desigualdad - diferencias culturales, de género, de raza y de etnia-, que no se agota en el estudio de la posición que el individuo ocupa en la división del trabajo.

$\mathrm{Al}$ entender la desigualdad como un concepto multidimensional, la hipótesis de partida apunta a incorporar la dimensión del tiempo al estudio de esta problemática. Considerando la disposición del tiempo como un recurso que permite el desarrollo personal y social de las personas, se puede afirmar la pertinencia de analizar las posibilidades de disponer $y$ organizar el tiempo "propio" como una forma de expresión de condiciones de desigualdad.

A partir de estas consideraciones, el análisis se centrará en las formas de organización del tiempo que despliegan las personas en su vida cotidiana. La hipótesis planteada exige un acercamiento que permita captar la mayor diversidad de situaciones que se dan en lo que se llama el mundo del trabajo; término que desde nuestra perspectiva, comprende todas las formas de empleo por el capital y públicas, en todos sus niveles y categorías, pasando por los servicios personales, el autoempleo, hasta la ocupación en actividades, al menos en apariencia, superfluas para la producción (Grassi y Danani, 2009). Asimismo, interesa indagar los modos en que se articulan y organizan los distintos ámbitos de la vida social - el trabajo remunerado, la socialidad, la vida familiar, el trabajo doméstico o reproductivo y el tiempo libre o de ocio-, que no se consideran como esferas claramente delimitadas/diferenciadas, sino más bien como espacios que interactúan y se entrecruzan.

\section{PRECISIONES METODOLÓGICAS}

El universo de estudio definido para la realización de la presente investigación está constituido por trabajadores de Partidos del Gran Buenos Aires. Operacionalmente, se define a los trabajadores como aquellos que recibieron algún tipo de pago por su trabajo. La unidad de estudio es la frontera entre mundo de trabajo $y$ vida cotidiana de dichos trabajadores ${ }^{3}$.

3 Se adopta la definición propuesta por Saltalamacchia (2005), quién señala que "la unidad de estudio debe ser entendida como un 
Como instrumento de recolección de datos, se utilizó una encuesta realizada entre los meses de octubre y noviembre de 2009 a trabajadores mayores de 18 años, residentes en Partidos del Gran Buenos Aires. Se construyó una muestra polietápica de 600 casos, representativa de esta población. Esta fue estratificada ${ }^{4}$ teniendo en cuenta criterios de sexo, edad y aglomerado del Gran Buenos Aires ${ }^{5}$. El nivel de confianza utilizado fue del orden del 95\%, con un margen de error de $+/-4,1 \%$.

\section{1. ÍNDICE DE LABILIDAD DE LA FRONTERA ENTRE VIDA COTIDIANA Y EL MUNDO DEL TRABAJO}

El propósito de la investigación realizada fue analizar la forma en que se erigen y diluyen las fronteras entre trabajo $y$ vida. Con vistas a acercarse a ese objetivo se construyó el "Índice de labilidad de las fronteras entre el tiempo de la vida y el tiempo de trabajo". Según la Real Academia Española, el adjetivo lábil significa "Que resbala o se desliza fácilmente/Frágil, caduco, débil/Poco estable, poco firme en sus resoluciones/Dicho de un compuesto: inesta-

concepto y a ella se le deben atribuir todas las características de (y se deben guardar todas las precauciones que requiere) cualquier concepto; entre ellos la de ser cuidadosamente definida. [...] Una de las confusiones más frecuentes emerge por no diferenciar la "unidad de estudio" de las "unidades de interrogación u observación”. Por ejemplo, si se quiere estudiar "El discurso de las adolescentes embarazadas sobre las causas y consecuencias de su embarazo", las unidades de interrogación son ciertas adolescentes embarazadas, pero esa no es la unidad de estudio. Por el contrario, la unidad es el discurso o los tipos de discurso; pues no se pretende conocer a las entrevistadas en su peculiaridad, sino en tanto portadoras de uno $\mathrm{u}$ otro discurso sobre el tema; son estos los que se quiere estudiar (pp.35-36).

Los criterios fueron definidos con base en los datos extraídos de la Encuesta Permanente de Hogares de octubre de 2001 del INDEC.

Composición de los aglomerados del GBA. GBA1: San Isidro, Vicente López; GBA2: Avellaneda, La Matanza 1, 3 de Febrero, San Martín, Hurlingham, Ituzaingó y Morón; GBA3: Lanús, Quilmes, Lomas de Zamora, Berazategui y Alte. Brown; GBA4: Tigre, Malvinas, J.C. Paz, San Miguel, Moreno, Merlo, Matanza 2, Ezeiza, E. Echeverría, F. Varela y San Fernando. ble, que se transforma fácilmente en otro". En nuestro caso, puede aplicarse a las esferas del trabajo y de la reproducción, cuya separación rígida fue una característica fundamental de la organización del trabajo capitalista. Asimismo, la idea de "labilidad" de estas fronteras, brindó herramientas analíticas para pensar las relaciones entre trabajo $y$ vida cotidiana, con miras a explorar las distintas posibilidades de organización del tiempo y el modo en que estas dan cuenta de situaciones de desigualdad.

\subsubsection{DEFINICIÓN OPERACIONAL}

Para la construcción de este índice, se utilizaron las siguientes preguntas contenidas en un cuestionario estructurado. Los entrevistados debían señalar qué actividades y con qué frecuencia las realizaban ${ }^{6}$ :

a) En el horario habitual de trabajo, ¿usted hace alguna de las siguientes cosas?: asistir a reuniones de padres del colegio de sus hijos, comunicarse con su casa para consultar cómo van las cosas, saber de sus hijos, organizar o dar indicaciones acerca de la comida, compras, etc., ir al médico o acompañar a su familia al médico, hablar con sus amigos por chat, teléfono, etc., leer cosas que le interesan, consultar internet por cuestiones no laborales, hacer trámites de la casa y estudiar.

b) Durante su horario laboral, ¿a usted lo llaman?: desde la escuela de sus hijos, desde el geriátrico, desde su casa, su cónyuge, otros familiares o amigos $^{7}$.

6 A las frecuencias, se les asignan los siguientes valores: siempre (4), frecuentemente (3), algunas veces (2), raramente (1) y nunca (0).

7 Para la construcción del índice, en primer lugar, se decidió clasificar las tareas según el grado de dedicación (alta, media o baja). Se considera que las tareas de "alta dedicación" implican tener que dejar el ámbito laboral y/o ser interrumpido por comunicaciones de los familiares o amigos en el horario de trabajo. Entre ellas, se agrupan las siguientes: asistir a reuniones del colegio de los hijos, ir al médico/atender cuestiones de salud 


\section{2. ÍNDICE DE PARTICIPACIÓN DOMÉSTICA}

Este índice permitió medir las condiciones de participación doméstica de los entrevistados, entendidas como aquellas condiciones que señalan la posición que la persona ocupa en la división del trabajo doméstico. Estas condiciones se observan en la dedicación de tiempo y trabajo que se destina al cuidado del hogar, a través de las tareas vinculadas con la reproducción ${ }^{8}$. Usualmente estas tareas se encuentran desigualmente distribuidas al interior de los hogares y recaen principalmente en las mujeres ${ }^{9}$. Se considera

propia o de su familia, hacer trámites de la casa y todas las llamadas recibidas en el horario laboral (desde la escuela de sus hijos, el geriátrico, su casa, llamadas del cónyuge y de otros familiares o amigos). Las tareas con "dedicación media" comportan un grado de obligatoriedad, pero pueden posponerse. Se consideran las siguientes: llamar a la casa para ver cómo van las cosas en general, saber de sus hijos, organizar o dar indicaciones acerca de la comida o compras y estudiar en el trabajo o hacer cosas vinculadas al estudio. Por último, las tareas de "baja dedicación", conllevan un cierto grado de gratificación y están vinculadas a la vida social y/o recreación, entre ellas, hablar con los amigos, leer cosas que interesen y consultar cuestiones no laborales en internet. En cuanto a los criterios de puntuación de estas actividades, se tomó como máximo posible, aquellos casos en que el entrevistado realizara todas las tareas mencionadas $y$ recibiera llamados siempre. Esta sumatoria da 700 puntos. Considerando este valor como máximo, se calculó el nivel de labilidad de las fronteras entre la vida cotidiana y el mundo del trabajo, $y$ se definieron las siguientes categorías: 1) Separación muy lábil, compuesta por aquellos casos que suman 300 o más puntos y 2) Separación poco lábil, los casos con 299 puntos $y$ menos.

Teniendo en cuenta las diferencias entre el carácter, el tiempo, la carga y el compromiso afectivo que implican el trabajo doméstico y las tareas vinculadas al cuidado, se decidió analizarlas separadamente. Por ello y como este artículo presenta los primeros resultados de una investigación en curso, para elaborar este índice de participación doméstica, se excluyen las tareas de "cuidado" (ocuparse de los niños u otros dependientes), que sí tendremos en cuenta en futuras aproximaciones, a medida que se vaya profundizando el análisis de la relación trabajo-vida cotidiana.

9 "Existe abundante evidencia de que la incorporación de la mujer al mercado laboral no implica un que las diferentes condiciones de participación doméstica, condicionan los modos de organizar el tiempo de trabajo y el tiempo de la vida.

\subsubsection{DEFINICIÓN OPERACIONAL}

Para construir el "índice de participación doméstica”, que permitió acceder a las diversas posiciones que ocupan los sujetos en el hogar y el tiempo que le dedican a las tareas vinculadas con la reproducción, se trabajó sobre la siguiente pregunta del cuestionario estructurado utilizado:

a) En su casa usted: prepara la comida, se ocupa de la ropa de la familia, limpia la casa, hace las compras, lava los platos, pone la mesa, hace la cama, hace reparaciones, paga las cuentas/hace trámites.

El encuestado debía seleccionar las diferentes opciones y señalar la frecuencia con la que realizaba dichas tareas.

Para ponderar las tareas, en primer lugar, se discutió la separación de las mismas en una clasificación de "pesadas", "livianas" o "intermedias". Las tareas pesadas responden a tres criterios: a) no pueden dejar de hacerse; b) implican una alta carga de trabajo $y$ tiempo, c) se hacen todos los días y de ellas depende el funcionamiento cotidiano de la casa. Se consideraron las siguientes: preparar la comida, ocuparse de la ropa de la familia y limpiar la casa. Las tareas intermedias conllevan una menor carga de trabajo y son: hacer las compras y lavar los platos. Por último, se consideran tareas livianas, aquellas que a) conllevan poco tiempo $y$ carga de trabajo $y$ b) que en algunos casos pueden posponerse. Entre ellas seleccionamos: poner la mesa, hacer la

aumento en el reparto entre varones y mujeres de la responsabilidad principal en la realización de las tareas reproductivas. Por el contrario, el reparto de esas responsabilidades se mantiene en una línea tradicional: las mujeres asumen este rol en mucho mayor medida que los varones $y$, en consecuencia, le destinan más tiempo" (Gherardi y Zibecchi, 2010: 15). 
cama, hacer reparaciones y pagar las cuentas/hacer trámites ${ }^{10}$.

\section{TRABAJO, TIEMPO Y DESIGUALDAD}

\subsection{LA LABILIDAD DE LOS LÍMITES}

Se presupone que la singular relación con el tiempo que establecen los sujetos, es expresión del modo en que se organiza la relación entre el trabajo y la vida cotidiana, organización que manifiesta múltiples tensiones relativas, al modo en que ambas esferas se recomponen en diferentes contextos de realización. Dicho en otros términos, la observación de la construcción social del tiempo que involucra a diversos grupos sociales, habilita un modo de entrada privilegiado para el estudio de la configuración de la relación entre trabajo y vida, y su relación con la reproducción de desigualdades y la aparición de nuevas expresiones de ella. Se profundizará en el análisis de una de las caras de la moneda de esta relación entre tiempo de trabajo y tiempo de vida: el modo en que el tiempo de trabajo es permeado por el tiempo de la vida social, familiar y doméstica.

10 En cuanto a los criterios de puntuación de las tareas, se tuvo en cuenta tanto la carga de las mismas, como su frecuencia. Se tomó como máximo posible, aquellos casos que hacen todas las actividades (pesadas, intermedias, livianas) siempre. Esta suma equivale a 340 puntos y el rango construido para considerar una participación doméstica alta fue entre los 250 y 340 puntos en la suma total de tareas. Se define una participación doméstica media como equivalente a aquellos casos en que los individuos hacen todas las tareas (pesadas, intermedias, livianas) frecuentemente o algunas veces (esta suma da 250 puntos). El rango de participación media construido, fue entre 125 y 249 puntos. Por último, se tomó como participación doméstica baja aquellos casos que hacen todas las tareas raramente o nunca (125 puntos) y el rango construido fue entre 0 y 124 puntos. El índice en cada uno de los casos, es la suma de todas las tareas que el entrevistado contestó que realiza en su frecuencia correspondiente. Cada caso entonces, tiene un número total de puntaje que permite ubicarlo en su "nivel de participación doméstica", según los criterios anteriormente explicados.
La labilidad de las fronteras entre estas dos esferas no es uniforme para todos los trabajadores. Cuestiones tales como el tipo de trabajo, las calificaciones, el lugar dónde este se desarrolla, el acceso a tecnologías de comunicación durante la jornada laboral o el género, condicionan, dificultan, permiten $y / 0$ facilitan la posibilidad de utilizar parte del tiempo formalmente dedicado al trabajo para ocuparse de cuestiones domésticas o sociales.

\section{GRÁFICO 1 \\ LABILIDAD ENTRE EL MUNDO DE LA VIDA Y EL MUNDO DEL TRABAJO}

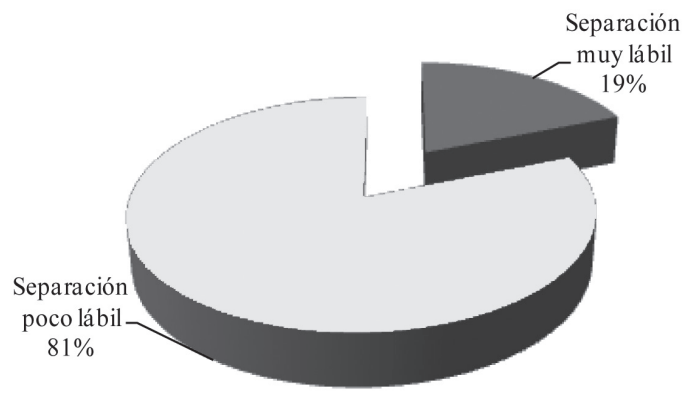

Fuente: Elaboración propia con base en datos de la encuesta "Condiciones de trabajo y de vida en Partidos del GBA". Octubre y noviembre de 2009.

En el gráfico 1, se observa que la mayoría de los entrevistados muestran una separación poco lábil entre su trabajo y su vida: ocho de cada diez, se encuentran en esta situación. Si bien, existe una extensa literatura que analiza las profundas transformaciones de las fronteras entre estas esferas $y$ el modo en que estas se vuelven cada vez más porosas, esta cifra muestra que si bien, efectivamente existen nuevas modalidades de organización del trabajo que hacen más porosas las fronteras entre el trabajo $y$ la vida social, familiar $y$ doméstica, estas no son generalizadas en todos los ámbitos laborales, primando aparentemente aún modalidades en las que las fronteras son poco o muy poco permeables. Cabe preguntarse, entonces, cuáles son los factores que inciden en la labilidad de esas fronteras. Sin duda, el género aparece como una variable ineludible para este análisis.

Si se considera la variable sexo, se observa que entre las mujeres aumenta el porcentaje de fronteras muy lábiles: $22,4 \%$ frente a un 
CUADRO 1

LABILIDAD DE LAS FRONTERAS ENTRE TIEMPO DE VIDA Y TIEMPO DE TRABAJO POR SEXO

\begin{tabular}{lccc}
\hline & FEMENINO & MASCULINO & TOTAL \\
\hline Fronteras muy lábiles & $22,4 \%$ & $15,7 \%$ & $19 \%$ \\
Fronteras poco lábil & $77,6 \%$ & $84,3 \%$ & $81 \%$ \\
TOTAL & $100 \%$ & $100 \%$ & $100 \%$ \\
TOTAL EN ABSOLUTOS & 295 & 305 & 600 \\
\hline
\end{tabular}

Fuente: Elaboración propia con base en datos de la encuesta "Condiciones de trabajo y de vida en Partidos del GBA". Octubre y noviembre de 2009.

15,7\% en los varones. Si bien, las diferencias de género que se manifiestan parecen responder a la naturalización del rol de la mujer como responsable de lo doméstico, aún cuando no esté presente en el hogar, la diferencia observada en estos porcentajes no es tan significativa como podría esperarse.

\subsection{LABILIDAD Y CICLO DE VIDA}

El ciclo vital de las persona suele marcar una relación diferente con la vida fami- liar y doméstica y con la sociabilidad en general.

El cuadro 2, muestra que en los extremos de la pirámide etárea, el porcentaje de baja labilidad de las fronteras entre vida cotidiana $y$ el mundo del trabajo, es mayor. Entre los trabajadores de 25 a 39 años se da el mayor porcentaje de una separación muy lábil. Puede plantearse la hipótesis, según la cual esto corresponde con las obligaciones familiares, que suelen ser mayores en ese rango de edad, particularmente entre las mujeres.

CUADRO 2

LABILIDAD DE LAS FRONTERAS ENTRE TIEMPO DE VIDA Y TIEMPO DE TRABAJO POR RANGO DE EDAD

\begin{tabular}{lccccc}
\hline & 18 A 24 AÑOS & 25 A 39 AÑOS & 40 A 59 AÑOS & 55 AÑOS Y MÁS & TOTAL \\
\hline Fronteras muy lábiles & $7,8 \%$ & $26,2 \%$ & $20,4 \%$ & $10 \%$ & $19 \%$ \\
Fronteras poco lábiles & $92,2 \%$ & $73,8 \%$ & $79,6 \%$ & $90 \%$ & $81 \%$ \\
TOTAL & $100 \%$ & $100 \%$ & $100 \%$ & $100 \%$ & $100 \%$ \\
TOTAL EN ABSOLUTOS & 90 & 229 & 181 & 100 & 600 \\
\hline
\end{tabular}

Fuente: Elaboración propia con base en datos de la encuesta "Condiciones de trabajo y de vida en Partidos del GBA". Octubre y noviembre de 2009.

Efectivamente, si se consideran las variables de sexo y edad de manera conjunta en el análisis de los ciclos de vida (cuadro 3), se observa que el mayor porcentaje de fronteras muy lábiles se da entre las mujeres de entre 25 y 39 años $(30,8 \%)$, mientras que el menor porcentaje se encuentra entre las mujeres mayores de 55 años (5,9\%). Entre los varones, los por- centajes de aquellos con fronteras menos rígidas son menores. Se puede presuponer que esta diferencia se explica, en parte, por las diferencias relacionadas con la participación doméstica de las mujeres (que se retoma más adelante), en general, mayor que la de los hombres y particularmente, entre las que cuidan hijos menores. 
CUADRO 3

LABILIDAD DE LAS FRONTERAS ENTRE TIEMPO DE VIDA Y TIEMPO DE TRABAJO POR RANGO DE EDAD Y SEXO

\begin{tabular}{|c|c|c|c|c|c|c|}
\hline & & 18 A 24 AÑOS & 25 A 39 AÑOS & 40 A 59 AÑOS & 55 AÑOS Y MÁS & TOTAL \\
\hline \multirow{4}{*}{ FEMENINO } & Fronteras muy lábiles & $9,5 \%$ & $30,8 \%$ & $26,8 \%$ & $5,9 \%$ & $22,4 \%$ \\
\hline & Fronteras poco lábiles & $90,5 \%$ & $69,2 \%$ & $73,2 \%$ & $94,1 \%$ & $77,6 \%$ \\
\hline & TOTAL & $100 \%$ & $100 \%$ & $100 \%$ & $100 \%$ & $100 \%$ \\
\hline & TOTAL (ABSOLUTOS) & 42 & 120 & 82 & 51 & 295 \\
\hline \multirow{4}{*}{ MASCULINO } & Fronteras muy lábiles & $6,3 \%$ & $21,1 \%$ & $15,2 \%$ & $14,3 \%$ & $15,7 \%$ \\
\hline & Fronteras poco lábiles & $93,8 \%$ & $78,9 \%$ & $84,8 \%$ & $85,7 \%$ & $84,3 \%$ \\
\hline & TOTAL & $100 \%$ & $100 \%$ & $100 \%$ & $100 \%$ & $100 \%$ \\
\hline & TOTAL (ABSOLUTOS) & 48 & 109 & 99 & 49 & 305 \\
\hline
\end{tabular}

Fuente: Elaboración propia con base en datos de la encuesta "Condiciones de trabajo y de vida en Partidos del GBA". Octubre y noviembre de 2009.

Cuando se toma en cuenta la composición del grupo familiar, se puede observar que la relación entre la labilidad de la frontera entre vida y trabajo está estrechamente vinculada al ciclo vital de las personas. El cuadro 4, muestra que aquellos trabajadores que tienen hijos (vivan con su pareja o solamente con sus hijos), son quienes presentan los mayores porcentajes de una separación muy lábil de las fronteras. En cambio, aquellos que viven solos, con su familia de origen, familia extendida o solamente con su pareja, tienen un alto porcentaje de fronteras poco lábiles (más del 80\%).

Cabe aclarar que el caso de los que viven solos presenta una situación particular en lo que refiere a la organización del tiempo, ya que la separación poco lábil entre trabajo y vida, puede estar relacionada, tanto con las características del empleo, como con la menor necesidad relativa de resolver situaciones vinculadas con la vida familiar o doméstica durante el tiempo dedicado al trabajo. De hecho, la totalidad de trabajadores de la muestra que viven solos, exhiben fronteras poco lábiles. 


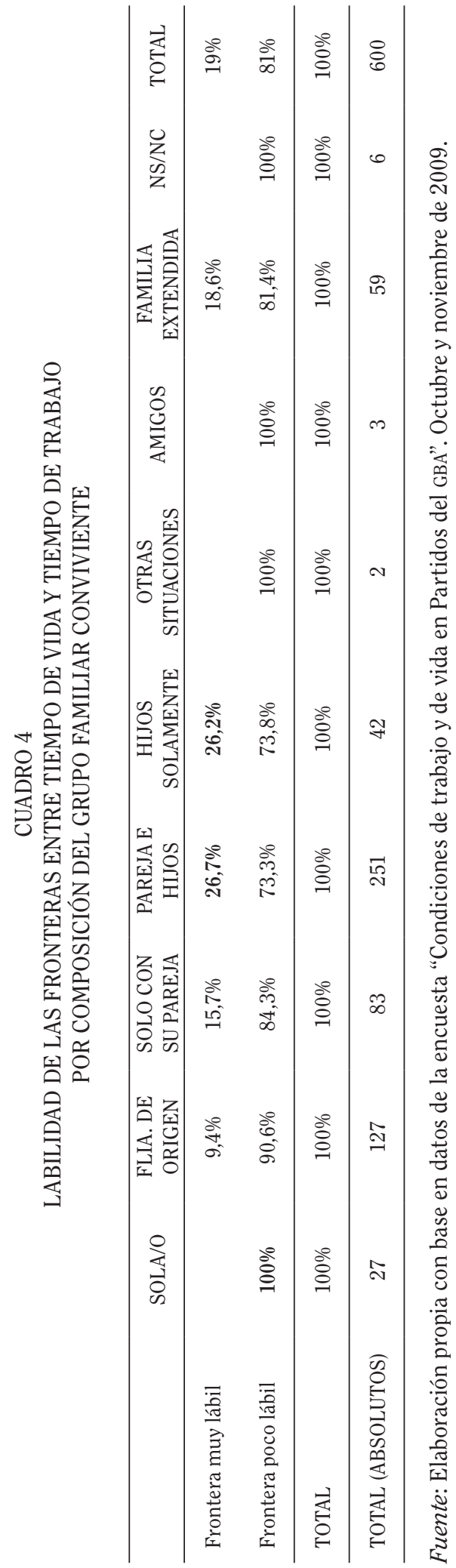




\subsection{LABILIDAD Y FORMAS DE INSERCIÓN LABORAL}

Los datos analizados, muestran una relación entre labilidad de las fronteras, el género $y$ el ciclo vital. Pero se debe considerar también, la situación laboral de los encuestados para comprender los modos de construcción de estos límites. El análisis de las diferentes condiciones de trabajo, muestra que estas juegan un rol fundamental en la determinación de la forma en que se definen esas fronteras.

CUADRO 5

LABILIDAD DE LAS FRONTERAS ENTRE TIEMPO DE VIDA Y TIEMPO DE TRABAJO POR EL TIPO DE TRABAJO

\begin{tabular}{|c|c|c|c|c|}
\hline & $\begin{array}{c}\text { FRONTERAS } \\
\text { MUY } \\
\text { LÁBILES }\end{array}$ & $\begin{array}{c}\text { FRONTERAS } \\
\text { POCO } \\
\text { LÁBILES }\end{array}$ & TOTAL & $\begin{array}{c}\text { TOTAL } \\
\text { ABSOLUTOS }\end{array}$ \\
\hline $\begin{array}{l}\text { Propietario de comercio o empresa/funcionarios/ } \\
\text { gerentes/jefes }\end{array}$ & $27,9 \%$ & $72,1 \%$ & $100 \%$ & 43 \\
\hline Empleados de oficina/comercio/cadetes & $21,4 \%$ & $78,6 \%$ & $100 \%$ & 117 \\
\hline Docentes/auxiliares de jardín/celadores & $26,5 \%$ & $73,5 \%$ & $100 \%$ & 34 \\
\hline Pequeños comerciantes/comercio familiar & $21,6 \%$ & $78,4 \%$ & $100 \%$ & 37 \\
\hline Profesionales & $35,7 \%$ & $64,3 \%$ & $100 \%$ & 14 \\
\hline Técnicos calificados & $27,6 \%$ & $72,4 \%$ & $100 \%$ & 29 \\
\hline $\begin{array}{l}\text { Operarios/peones/mantenimiento/obrero/maestranza/ } \\
\text { gastronómicos (mozos, cocineros) }\end{array}$ & $6,4 \%$ & $93,6 \%$ & $100 \%$ & 109 \\
\hline Oficios con calificación & $16,4 \%$ & $83,6 \%$ & $100 \%$ & 61 \\
\hline Empleados de seguridad & $18,8 \%$ & $81,3 \%$ & $100 \%$ & 16 \\
\hline Comercio marginal/trabajadores manuales a destajo & $27,3 \%$ & $72,7 \%$ & $100 \%$ & 33 \\
\hline $\begin{array}{l}\text { Servicio doméstico/limpieza no doméstica/cuidado de } \\
\text { niños y enfermos en o a domicilio }\end{array}$ & $13,1 \%$ & $86,9 \%$ & $100 \%$ & 61 \\
\hline $\begin{array}{l}\text { Artesanos/artistas/profesores de manualidades y artes/ } \\
\text { servicios personales, de belleza/New Age }\end{array}$ & $25 \%$ & $75 \%$ & $100 \%$ & 24 \\
\hline Choferes & $18,2 \%$ & $81,8 \%$ & $100 \%$ & 22 \\
\hline & \multicolumn{2}{|c|}{ PROMEDIO } & \multicolumn{2}{|c|}{ TOTAL } \\
\hline & $19 \%$ & $81 \%$ & $100 \%$ & 600 \\
\hline
\end{tabular}

Fuente: Elaboración propia con base en datos de la encuesta "Condiciones de trabajo y de vida en Partidos del GBA". Octubre y noviembre de 2009. 
Si se considera el tipo de actividad que realizan las personas encuestadas, en el cuadro 5 se observa que entre los trabajos menos calificados se dan las condiciones de mayor rigidez de las fronteras entre trabajo y vida cotidiana. En la categoría de "operarios/peones/mantenimiento/obreros/maestranza/gastronómicos", la separación poco lábil de las fronteras llega al 93,6\%. A estos le siguen los trabajadores del servicio doméstico, limpieza en general o tareas de cuidado de niños, ancianos o enfermos, con un $86,9 \%$. Cabe destacar que este sector está compuesto en su totalidad por mujeres, como se muestra en el cuadro 6.

En oposición, entre los trabajadores profesionales, se da el porcentaje más alto de situaciones de fronteras muy lábiles entre ambas esferas: el 35,7\% tienen trabajos que les permiten resolver sus situaciones de la vida social, familiar y doméstica durante el tiempo dedicado formalmente al trabajo. Esta categoría es seguida por los "propietario de comercio o empresa/funcionarios/gerentes/ jefes" con un $27,9 \%$.
Si se observa los casos de trabajadores dedicados al "comercio marginal/trabajadores manuales a destajo", ocupaciones caracterizadas por requerir una baja calificación y presentar mayormente situaciones laborales inestables, en condiciones de precarización y con bajos ingresos (Salvia et ál., 2008), se encuentran porcentajes de fronteras muy lábiles que se acercan a los de las categorías más calificadas mencionadas antes. Tomando la variable género incorporada en el cuadro 6 , se puede observar que dentro de este grupo, 7 de cada 10 trabajadores son mujeres.

Se puede retomar el análisis realizado en el punto 4.2 acerca de la relación entre ciclo de vida, organización del tiempo y género, considerando como las categorías de "comercio marginal/trabajadores manuales a destajo" y "docentes o auxiliares", tareas realizadas mayormente por mujeres, aparecen como trabajos caracterizados por presentar fronteras muy lábiles entre trabajo $y$ vida, que salvo estas dos excepciones, se presenta en los trabajos más calificados $y / 0$ de mejores ingresos, como se verá en el apartado 4.4 . 


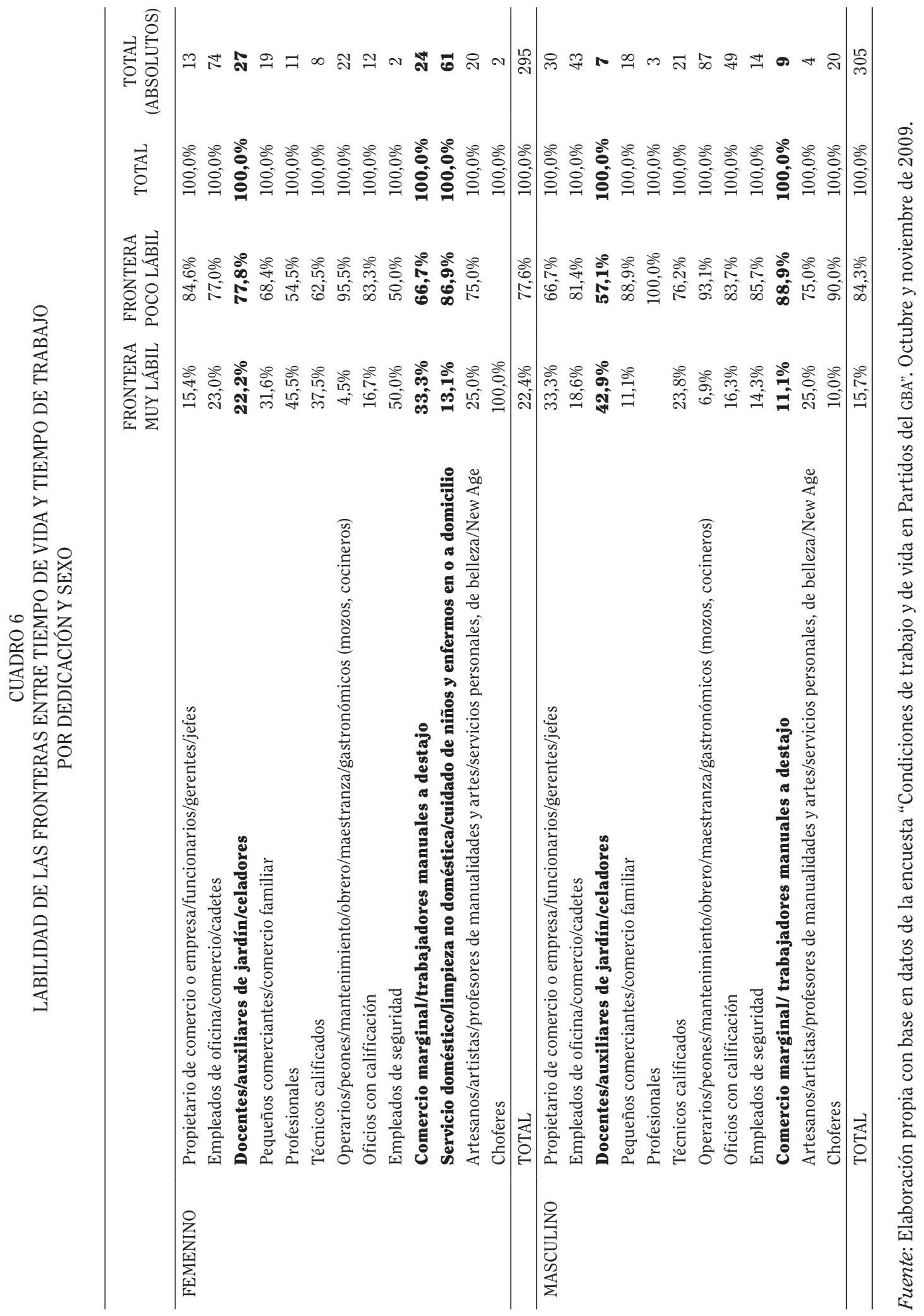


Analizando los datos presentados en los cuadros 5 y 6 , desde la perspectiva de análisis de la desigualdad, se observa que por un lado, se encuentran aquellas mujeres que trabajan en el servicio doméstico o se dedican a tareas de cuidado, quienes presentan las fronteras más rígidas en la organización del tiempo de trabajo y el tiempo de la vida social, familiar y doméstica. Por otro, tenemos un grupo de mujeres ocupadas en trabajos igualmente poco calificados (trabajo marginal y manual) o de bajos ingresos (docentes y auxiliares), pero cuyas fronteras entre trabajo y vida son más lábiles. Esta posibilidad en la organización del tiempo, las ubica en una posición más favorable respecto a aquellas mujeres cuyos trabajos imposibilitan una organización más flexible para responder a sus obli- gaciones y necesidades domésticas. Si se consideran las formas de producción y reproducción de la desigualdad, aparece en primera instancia la cuestión de la segmentación del mercado de trabajo por género, vinculada también al hecho de que son las mujeres quienes tienen en mayor medida peores empleos $y$ menos remunerados (Con et ál., 2003 y Castillo et ál., 2008). Sin embargo, al considerar la combinación de las variables ocupación, calificación y organización del tiempo, se observa que al interior de este grupo de mujeres, existen distintas situaciones que expresan condiciones desiguales "invisibles", si se piensa exclusivamente en relación con las condiciones que presentan frente a los varones. Se trata de desigualdades más difíciles de medir y por ello menos perceptibles.

CUADRO 7

LABILIDAD DE LAS FRONTERAS ENTRE TIEMPO DE VIDA Y TIEMPO DE TRABAJO POR LUGAR DONDE SE REALIZA EL TRABAJO

\begin{tabular}{lcccc}
\hline & FRONTERAS & FRONTERAS & TOTAL & $\begin{array}{c}\text { TOTAL } \\
\text { (ABSOLUTOS) }\end{array}$ \\
\hline EUY LÁBILES & POCO LÁBILES & & 74 \\
Oficina/organismo del Estado & $23 \%$ & $77 \%$ & $100 \%$ & 72 \\
Calle/venta a domicilio/chofer/medios de transporte & $24,1 \%$ & $75,9 \%$ & $100 \%$ & 58 \\
Si lugar fijo/depende del trabajo/depende del cliente & $8,1 \%$ & $91,9 \%$ & $100 \%$ & 86 \\
Fábrica/taller/comercio & $15,2 \%$ & $84,8 \%$ & $100 \%$ & 197 \\
Una casa ajena & $14,9 \%$ & $85,1 \%$ & $100 \%$ & 47 \\
Establecimiento educativo/de salud & $26,3 \%$ & $73,7 \%$ & $100 \%$ & 57 \\
Comedor/org. social & & $100 \%$ & $100 \%$ & 3 \\
Camping/club/barrio privado & $16,7 \%$ & $83,3 \%$ & $100 \%$ & 6 \\
\hline TOTAL & $19 \%$ & $81 \%$ & $100 \%$ & 600 \\
\hline
\end{tabular}

Fuente: Elaboración propia con base en datos de la encuesta "Condiciones de trabajo y de vida en Partidos del GBA". Octubre y noviembre de 2009.

En el análisis de la labilidad de los límites entre tiempo de trabajo $y$ de vida, el lugar dónde se realiza el trabajo juega también un rol importante. Aquellos que lo hacen sin lugar fijo, de acuerdo con las necesidades del cliente, son los que tienen menos posibilidades de utilizar su tiempo de trabajo para resolver cuestiones domésticas y/o sociales. En oposición, los oficinistas son quienes tienen los porcentajes mayores de una separación muy lábil.
En el caso de quienes trabajan en su propio domicilio, si bien, el porcentaje de alta labilidad se encuentra por encima del porcentaje general que es del 19\%, es llamativo que el porcentaje de quienes presentan fronteras poco lábiles sea elevado (77\%). Esto se explica en parte, porque en muchos casos los encuestados manifestaron que en realidad adaptan los horarios de trabajo a las cuestiones de la vida doméstica, por lo que no consideran que 
ambos tiempos se entremezclen, sino más bien, se da una subordinación del tiempo laboral al tiempo de vida.

\subsection{LABILIDAD Y NIVEL DE INSTRUCCIÓN}

El capital cultural (Bourdieu, 1988), traducido en titulaciones de los entrevistados, aparece como una de las variables más significativas en la construcción de la labilidad de las fronteras que se están analizando.

El cuadro 8 muestra que la posibilidad de obtener trabajos con mayor labilidad de las fronteras mundo de trabajo y de la vida, aumenta a medida que aumenta la calificación. De hecho, son los universitarios quienes tienen un mayor porcentaje de trabajadores en esta categoría, mientras que entre aquellos sin instrucción o con primaria incompleta, se ubica el menor porcentaje.

\section{CUADRO 8}

LABILIDAD DE LAS FRONTERAS ENTRE TIEMPO DE VIDA Y TIEMPO DE TRABAJO POR NIVEL DE INSTRUCCIÓN

\begin{tabular}{lccccccc}
\hline & $\begin{array}{c}\text { SIN INS- } \\
\text { TRUCCIÓN/ } \\
\text { PRIMARIA } \\
\text { INCOMPLETA }\end{array}$ & $\begin{array}{c}\text { PRIMARIA } \\
\text { COMPLETA/ } \\
\text { SECUN- } \\
\text { DARIA } \\
\text { INCOMPLETA }\end{array}$ & $\begin{array}{c}\text { SECUN- } \\
\text { DARIA } \\
\text { COMPLTA }\end{array}$ & $\begin{array}{c}\text { TERCIARIA } \\
\text { O } \\
\text { UNIVE- } \\
\text { SITARIA } \\
\text { INCOMPLETA }\end{array}$ & $\begin{array}{c}\text { TERCIARIA } \\
\text { COMPLETA }\end{array}$ & $\begin{array}{c}\text { UNIVER- } \\
\text { SITARIA } \\
\text { C MÁS }\end{array}$ & TOTAL \\
\hline Frontera muy lábil & $11,5 \%$ & $15,1 \%$ & $19,3 \%$ & $24,6 \%$ & $26,3 \%$ & $37,9 \%$ & $19 \%$ \\
Frontera poco lábil & $88,5 \%$ & $84,9 \%$ & $80,7 \%$ & $75,4 \%$ & $73,7 \%$ & $62,1 \%$ & $81 \%$ \\
\hline TOTAL & $100 \%$ & $100 \%$ & $100 \%$ & $100 \%$ & $100 \%$ & $100 \%$ & $100 \%$ \\
\hline TOTAL (ABSOLUTOS) & 26 & 259 & 187 & 61 & 38 & 29 & 600 \\
\hline
\end{tabular}

Fuente: Elaboración propia con base en datos de la encuesta "Condiciones de trabajo y de vida en Partidos del GBA". Octubre y noviembre de 2009.

Estos datos dan cuenta de una relación fuerte $y$ progresiva entre el nivel educativo y las posibilidades de una organización del tiempo de trabajo y de vida más flexible. Esto permite plantear dos hipótesis: a) aquellos que tienen trabajos que requieren mayores credenciales educativas, tienen más posibilidades de acceso a trabajos cuyas fronteras son más lábiles y b) los empleos que permiten "filtrar" tiempo dedicado a actividades vinculadas con la vida cotidiana, parecen ser los más valorados, ya que quienes cuentan con márgenes mayores en la elección de las condiciones o tipos de empleo (los más calificados), procuran hacerlo en aquellos trabajos, cuyas fronteras son más lábiles. 


\subsection{TRABAJO Y CONDICIONES DE PARTICIPACIÓN DOMÉSTICA: LA CUESTIÓN DE LA DOBLE JORNADA LABORAL}

Como se ha visto, la labilidad de las fronteras entre el tiempo de la vida y el tiempo de trabajo no guarda necesariamente relación con las obligaciones domésticas de los encuestados, ya que una frontera poco lábil significa la imposibilidad de distraer tiempo de trabajo para cuestiones vinculadas con la vida social, familiar y doméstica, pero nada dice acerca del volumen de tareas reproductivas que se realizan y el tiempo que estas demandan.

\section{GRÁFICO 2 \\ PARTICIPACIÓN DOMÉSTICA POR LABILIDAD \\ ENTRE VIDA COTIDIANA Y MUNDO DEL TRABAJO}

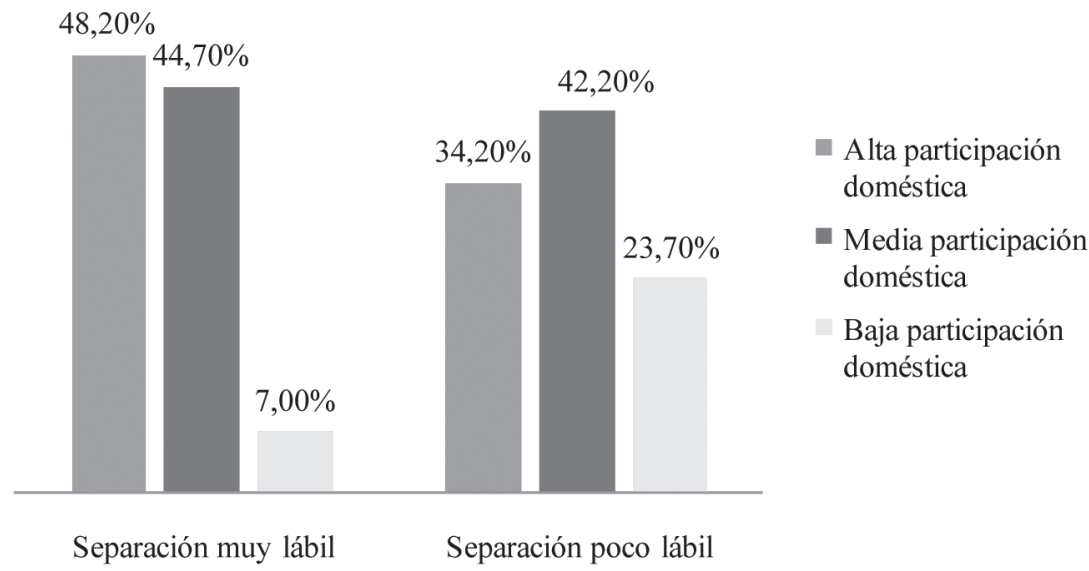

Fuente: Elaboración propia con base en datos de la encuesta "Condiciones de trabajo y de vida en Partidos del GBA". Octubre y noviembre de 2009.

El gráfico 2 muestra que un $34,2 \%$ de los entrevistados que tienen una alta participación doméstica, tienen también fronteras poco lábiles entre tiempo de trabajo y de vida. En números absolutos, se trata de 166 casos (cuadro 9).
Esta situación da cuenta de que estas personas deben hacerse cargo de las tareas vinculadas a la reproducción, totalmente por fuera del tiempo de trabajo; es decir, que viven una situación de sobrecarga.

CUADRO 9

SEXO DE LOS ENTREVISTADOS CON ALTA PARTICIPACIÓN DOMÉSTICA Y FRONTERAS POCO LÁBILES

\begin{tabular}{ccc}
\hline & CASOS & PORCENTAJE \\
\hline Femenino & 133 & 80,1 \\
Masculino & 33 & 19,9 \\
TOTAL & 166 & 100,0 \\
\hline
\end{tabular}

Fuente: Elaboración propia con base en datos de la encuesta "Condiciones de trabajo y de vida en Partidos del GBA". Octubre y noviembre de 2009. 
Si se considera la variable sexo, de quienes tienen alta participación doméstica y una frontera poco lábil entre trabajo y vida, se comprueba que el $80,1 \%$ de estos casos son mujeres. Aquí aparece una situación de desigualdad de género que no se observaba tan claramente en el cuadro 1 , cuando se analizó la labilidad de las fronteras entre vida cotidiana y el mundo del trabajo por sexo del entrevistado para la totalidad de la muestra. Este dato no sorprende. Existen numerosos estudios (Murillo, 1996; Comas D’Argemir, 2000; Pautassi, 2007; Batthyány, 2007 y Aguirre, 2007) que analizan la construcción tradicional de los roles de género en la división sexual del trabajo y que ubican a la mujer como responsable del hogar, del cuidado $y$ de las tareas domésticas, $y$ al varón como proveedor de los ingresos provenientes del trabajo remunerado.

En este sentido, el aumento sostenido de la incorporación de la mujer al mercado de trabajo a partir de la década de 1980 y más fuertemente en la década de 1990, no estuvo acompañado en la misma medida, de la transformación de los roles de género tradicionales, ni de la co-responsabilidad de varones $y$ mujeres en el trabajo doméstico. Esta situación da lugar al fenómeno que se denominó como "doble jornada", aludiendo al trabajo que la mujer despliega tanto en la esfera productiva como en la reproductiva (Pautassi, 2007; Gherardi y Zibecchi, 2010). Esta situación de "doble jornada" que muestran los datos de los cuadros anteriores, se profundiza si se considera que el $45 \%$ de estas trabajadoras labora más de 7 horas (cuadro 10).

CUADRO 10

CANTIDAD DE HORAS QUE TRABAJAN QUIENES TIENEN ALTA PARTICIPACIÓN DOMÉSTICA Y FRONTERAS POCO LÁBILES ENTRE TIEMPO DE VIDA Y TIEMPO DE TRABAJO

\begin{tabular}{ccc}
\hline & CASOS & PORCENTAJE \\
\hline Hasta 4 horas & 29 & 17,5 \\
Entre 5 y 6 horas & 34 & 20,5 \\
Entre 7 y 9 horas & 65 & 39,2 \\
Entre 10 y 12 horas & 31 & 18,7 \\
13 horas $y$ más & 4 & 2,4 \\
Sin un horario fijo & 3 & 1,8 \\
TOTAL & 166 & 100,0 \\
\hline
\end{tabular}

Fuente: Elaboración propia con base en datos de la encuesta "Condiciones de trabajo y de vida en Partidos del GBA". Octubre y noviembre de 2009.

\section{CONCLUSIONES}

El objetivo de este artículo fue analizar los modos en que se constituyen relaciones desiguales a través de la organización del tiempo, según las distintas formas de inserción laboral $y$ las condiciones de participación doméstica de las personas. Particularmente, se centró en indagar la forma en que la vida social, familiar $y$ doméstica se "filtra" en el tiempo formalmente dedicado a las actividades laborales.
En cuanto a los resultados, la investigación realizada mostró que si bien, estas dos esferas de la vida social -históricamente construidas como espacios claramente delimitados - se encontrarían en un proceso de reconfiguración, debido a las transformaciones en los medios, organización y gestión del trabajo que favorecen un mayor entrecruzamiento entre esos ámbitos y una mayor porosidad de sus fronteras. Se halla que en el contexto que 
representa la muestra, prima aún una forma de organización del tiempo en la que las fronteras entre el tiempo de trabajo y el tiempo de la vida son considerablemente rígidas.

No obstante, el trazado de esos límites y las oportunidades de realización de actividades vinculadas a la vida social, familiar y doméstica en el espacio y el tiempo de trabajo, se encuentran desigualmente distribuidas. La posibilidad de "filtrar" tiempo para actividades vinculadas con la reproducción y la socialidad, durante el tiempo formalmente destinado al trabajo, se da de distintos modos y con diferentes intensidades en cada sector social y según las diversas posiciones laborales de los individuos. Los datos analizados permiten afirmar que estas posibilidades se encuentran condicionadas fuertemente por las características del trabajo que se realiza (lugar dónde desarrollan las tareas, tipo de tareas, etc.) junto a las calificaciones $y$ titulaciones de los trabajadores.

Se puede afirmar que existe una relación progresiva entre el nivel educativo alcanzado y las posibilidades de una organización del tiempo más flexible. Esta situación lleva a reflexionar acerca de las posibilidades de acceso a distintos empleos y las elecciones que pueden realizar los sujetos: en el caso de aquellos que cuentan con mayores calificaciones, se observa que estos acceden a cierto tipo de trabajos que les permiten "filtrar" tiempo de la vida en la jornada formalmente dedicada al trabajo. De modo opuesto, son los trabajadores que poseen menos credenciales educativas, quienes tienen trabajos que les permiten menores posibilidades de realizar esos arreglos, por ello deben ocuparse de dichas cuestiones completamente por fuera del horario laboral.

En la consideración de las características de estos límites, quienes trabajan en su domicilio presentan un caso particular, ya que esa pareciera ser la situación dónde las fronteras muestran la mayor labilidad e indiferenciación. Se quisiera dejar planteado, a modo de hipótesis, que en estos casos, las necesidades de la vida podrían estar funcionando como organizadoras del trabajo (y no viceversa, como se podría afirmar para la mayor parte de las ocupaciones).
El índice de labilidad de las fronteras entre tiempo de trabajo y tiempo de vida, permitió ver que las mujeres son las que tienen porcentajes más altos de fronteras muy lábiles entre ambas esferas, sumando el mayor porcentaje en el caso de las mujeres de 25 a 39 años. Esta situación se vincula, por un lado, con el ciclo vital de las personas, dado que es en esa franja etárea, cuando los sujetos tienen mayores responsabilidades domésticas y de cuidado, vinculadas con la crianza y la atención de los niños. En este sentido, es también en este rango de edad en el que los varones presentan los mayores índices de fronteras muy lábiles entre el mundo del trabajo y la vida. A pesar de ello, los datos presentados parecen indicar que las mujeres trabajadoras mantienen el rol tradicional de sostener las obligaciones domésticas.

Cuando se analiza la relación entre fronteras poco lábiles y alta dedicación doméstica, se comprueba que poco más de tres de cada diez trabajadores está en esa situación y de ese porcentaje, el 80,1\% son mujeres. Estos casos presentan la situación que probablemente sea la más desfavorable en la organización del tiempo, ya que las fronteras del tiempo de trabajo se corresponden a un modelo tradicional, con una clara delimitación y gran rigidez, a la vez que no pueden delegar las obligaciones domésticas. En cuanto al tipo de trabajo que realiza este grupo, un alto porcentaje se dedica a tareas poco calificadas y tiene también una carga horaria de trabajo importante.

Si las posibilidades de disposición del tiempo constituyen una dimensión de la desigualdad social, las formas en que se relacionan tiempo de trabajo $y$ tiempo de vida, dan cuenta de esta. Lo que muestra la conformación de este grupo, es precisamente cómo se (re)produce la desigualdad a través de la distribución del tiempo y la posibilidad que la permeabilidad de las fronteras da a aquellos que pueden utilizar parte de su tiempo de trabajo para resolver cuestiones de la vida social y doméstica, frente a quienes ven sobrecargada su jornada debido precisamente a la rigidez de esas fronteras. 
Esta fue una primera aproximación que aportó algunos elementos para el análisis de la compleja problemática de la desigualdad y el trabajo en el conurbano bonaerense. Se considera que la profundización del estudio de la construcción social de los límites entre tiempo de trabajo y de vida, contribuirá al análisis del modo en que esa relación con el tiempo expresa situaciones de desigualdad.

\section{BIBLIOGRAFÍA}

LIBROS

Aguirre, Rosario. "Trabajar y tener niños: insumos para repensar las responsabilidades familiares y sociales". Género, familias y trabajo: rupturas y continuidades. Desafios para la investigación política. Gutiérrez, María Alicia (ed.). Buenos Aires: ClACSO, 2007.

Aguirre, Rosario; García Sainz, Cristina y Carrasco, Cristina. "El tiempo, los tiempos, una vara de desigualdad". Serie Mujer y Desarrollo 65. Santiago de Chile: CEPAL, 2005.

Batthyani, Karina. "Articulación entre vida laboral $y$ vida familiar. Las prácticas de cuidado infantil de trabajadoras asalariadas de Montevideo". Género, familias y trabajo: rupturas $y$ continuidades. Desafíos para la investigación politica. Gutiérrez, María Alicia (comp.). Buenos Aires: Clacso, 2007.

Boltansky, Luc y Chiapello, Eve. El nuevo espíritu del capitalismo. España: Ediciones Akal, 2002.

Bourdieu, Pierre. "Espacio social y poder simbólico". Cosas dichas. Barcelona: Gedisa, 1988.

Castel, Robert. Trabajo y utilidad en el mundo. Buenos Aires: Topía Editorial, 2004.

Castillo, Victoria et ál. "Los efectos del nuevo patrón de crecimiento sobre el empleo femenino, 2003-2006". El trabajo femenino en la post convertibilidad. Argentina 2003-2007. Novick, Marta; Rojo, Sofia y Castillo, Victoria (comps.). Buenos Aires: MTESS, CEPAL, GTZ, 2008.
Comas, Dolores. "Mujeres, familia y Estado del Bienestar". Perspectivas feministas desde la antropología social. Del Valle, Teresa (ed.). Barcelona: Ariel Antropología, 2000.

Con, Melina; Epstein, Elisa; Pacetti, Ana y Salvia, Agustín. "Cambios en la estructura socio-ocupacional en el GBA durante los 90. Una mirada desde la problemática del género". VII Jornadas de Historia de las Mujeres y II Congreso Iberoamericano de Estudios de Género, 2003.

Gherardi, Natalia y Zibecchi, Carla. "El derecho al trabajo y la ocupación de las mujeres: una visión regional para América Latina". Género y empleo: iniciativas de la sociedad civil como modelos para la construcción de políticas públicas de empleo con mirada de género. Di Pietro, María Paula (comp.). Buenos Aires: ELA, 2010. En: <http://www.ela.org.ar>

Grassi, Estela y Danani, Claudia (orgs.). El mundo del trabajo y los caminos de la vida. Trabajar para vivir; vivir para trabajar. Buenos Aires: Espacio, 2009.

Luci, Florencia. "Nuevas formas de trabajo en las grandes empresas: individualización y estrategias de carrera entre asalariados de altos puestos". El mundo del trabajo y los caminos de la vida. Trabajar para vivir; vivir para trabajar. Grassi, Estela y Danani, Claudia (orgs.). Buenos Aires: Espacio, 2009.

Pautassi, Laura. ¡Cuánto trabajo mujer! El género y las relaciones laborales. Buenos Aires: Capital Intelectual, 2007.

Saltalamacchia, Homero Rodolfo. Del proyecto al análisis: aportes a una investigación cualitativa socialmente útil. Buenos Aires: El autor, 2005.

Salvia, Agustín et ál. "Cambios en la estructura social del trabajo bajo los regímenes de convertibilidad y posdevaluación. Una mirada desde la perspectiva de la heterogeneidad estructural". Trabajo, ingresos y politicas en Argentina. Contribuciones para pensar el siglo XXI. 
Lindenboim, Javier (comp.). Buenos Aires: Eudeba, 2008.

Thompson, Edward. Tradición, revuelta y conciencia de clase. Barcelona: Crítica, 1989.

Wainerman, Catalina. "Conyugalidad y paternidad ¿Una revolución estancada?”. Género, familias y trabajo: rupturas y continuidades. Desafíos para la investigación politica. En: Gutiérrez, María Alicia. Buenos Aires: CLACso, 2007.

Fecha de ingreso: 29/11/2011

Fecha de aprobación: 10/02/2012 
